\title{
Hepatectomia parcial por adenoma gigante: um relato de caso
}

\author{
Partial hepatectomy for giant adenoma: a case report \\ Hepatectomía parcial por adenoma gigante: reporte de un caso
}

Verônica Maciel Atalla ${ }^{1 *}$, Adriana Rodrigues Ferraz ${ }^{1}$, Amanda Lúcia Arruda Pontes ${ }^{1}$, Breno Castro Corrêa de Figueiredo ${ }^{1}$, Izadora Souza Farias ${ }^{1}$, Milena Pereira Costa ${ }^{1}$, Paulo Víctor Innocencio Póvoa de Castro $^{1}$, Suzane Aguiar de Souza1, Thalles Vítor Teixeira Pacífico ${ }^{1}$, Yago Paranhos de Assis .

\section{RESUMO}

Objetivo: Elucidar aspectos diagnósticos e terapêuticos acerca do Adenoma Hepático a fim de realizar o diagnóstico precoce evitando complicações que possam ser fatais. Detalhamento do caso: Paciente feminino, 31 anos, usuária de Contraceptivo Oral Combinado (AOC) há anos, assintomática, realizou Ultrassonografia (USG) de abdome total de rotina, no qual foram evidenciados vários nódulos hepáticos, sendo o maior, um nódulo sólido isoecoico a levemente hipoecoico, de contorno regular, medindo $8,1 \times 7,1 \times$ $4,9 \mathrm{~cm}(\mathrm{~T} \times \mathrm{L} \times \mathrm{AP})$ localizado inferiormente ao segmento VI do fígado. Como terapêutica foi realizada segmentectomia hepática com posterior seguimento anual por Ressonância Magnética dos outros nódulos residuais, que permanecem estáveis. Considerações finais: O Adenoma Hepático é uma doença rara que pode ser diagnosticada de forma incidental, em mulheres assintomáticas bem como pode causar dor em hipocôndrio direito e deflagrar quadro de hemorragia intraperitoneal com posterior óbito. Portanto, é de suma importância seu reconhecimento e terapêutica precoce a fim de evitar essa complicação.

Palavras-chave: Adenoma hepático, Anticoncepcionais orais combinados, Hemorragia intratumoral.

\begin{abstract}
Objective: To elucidate diagnostic and therapeutic aspects about Hepatic Adenoma in order to make an early diagnosis avoiding complications that could be fatal. Detail of the case: Female patient, 31 years old, user of Combined Oral Contraceptive $(A O C)$ for years, asymptomatic, underwent routine total Abdomen Ultrasound (USG), in which several hepatic nodules were found, the largest being a solid isoechoic nodule. the slightly hypoechoic, of regular contour, measuring $8.1 \times 7.1 \times 4.9 \mathrm{~cm}(T \times W \times A P)$ located inferiorly to the VI segment of the liver. As therapy, liver segmentectomy was performed with subsequent annual follow-up by Magnetic Resonance os the other residual nodules, which remain stable. Final considerations: Hepatic adenoma is a rare disease that can be diagnosed incidentally, in asymptomatic women, as well as causing pain in the right hypochondrium and trigeering intraperitoneal hemorrhage with subsequent death. Therefore, recognition and early therapy is of utmost importance in order to avoid this complication.
\end{abstract}

Key words: Hepatic adenoma, Combined oral contraceptives, Intratumoral hemorrhage.

\section{RESUMEN}

Objetivo: Dilucidar los aspectos diagnósticos y terapéuticos del Adenoma Hepático para realizar un diagnóstico precoz evitando complicaciones que pudieran ser fatales. Detalle del caso: Paciente de sexo femenino, 31 años, usuaria de Anticonceptivo Oral Combinado (AOC) durante años, asintomática, sometida a Ecografía Total de Abdomen (USG) de rutina, en la que se encontraron varios nódulos hepáticos, siendo el

${ }^{1}$ Universidade de Vassouras, Vassouras - RJ. *E-mail: veronica_atallastrela@hotmail.com 
mayor un nódulo isoecoico sólido. el contorno regular, ligeramente hipoecoico, de 8,1 x 7,1 x 4,9 cm ( T x W x AP) situado debajo del segmento VI del hígado. Como terapia se realizó una segmentectomía hepática con posterior seguimiento anual mediante resonancia magnética de otros nódulos residuales, que permanecen estables. Consideraciones finales: El adenoma hepático es una enfermedad rara que puede diagnosicarse de manera incidental, en mujeres asintomáticas, además de causar dolor en el hipocondrio derecho y desencadenar una hemorragia intraperitoneal con posterior muerte. Por tanto, su reconocimiento y tratamiento precoz es de suma importancia para evitar esta compicación.

Palabras clave: Adenoma hepático, Anticonceptivos orales combinados, Hemorragia intratumoral.

\section{INTRODUÇÃO}

O aprimoramento dos exames de imagem e o crescente acesso a eles, permite, de forma exponencial, o diagnóstico de diversas afecções, entre elas, os tumores hepáticos benignos assintomáticos. Os tumores possuem características próprias que podem ser identificadas e analisadas para que se possa ser feito 0 diagnóstico de forma correta. Dentre eles, destacam-se o Hemangioma, a Hiperplasia Nodular Focal e o Adenoma Hepático (COELHO JCU, et al., 2011).

O Adenoma Hepático (AH) é um tumor sólido raro, que acomete cerca de 1:100.000 mulheres principalmente em idade fértil, solitário em $80 \%$ dos casos e localiza-se preferencialmente no lobo hepático direito (SZOR DJ, et al., 2013).

Nos últimos anos, houve elevação do número de casos tendo como associação o crescente uso de contraceptivo oral e esteroides anabolizantes. Dentre outros fatores de risco, destaca-se, a Polipose Adenomatosa Familiar (PAF), obesidade, glicogenoses e o consumo de álcool (STRAUSS E, et al., 2015).

As células formadoras do tumor frequentemente apresentam tamanho normal ou levemente aumentado, dispostas de forma irregular, contendo em seu interior depósitos de glicogênio e gotículas de gordura, a depender do seu subtipo (PINTO P, 2012).

O AH pode ser diagnosticado de forma incidental nos quadros assintomáticos em que são realizados exames de rotina, como Ultrassonografia (USG) de abdome. Além disso, essa patologia pode ocasionar quadros de dor abdominal em andar superior, principalmente do lado direito, hepatomegalia, compressão de estruturas adjacentes e como complicação pode romper e evoluir para um quadro de hemorragia intraperitoneal (PAIS-COSTA SR, et al., 2012). O sangramento se comporta como um quadro relativamente frequente e que possui altos índices de morbimortalidade (SZOR DJ, et al., 2013).

O mecanismo que relaciona o Anticoncepcional Oral Combinado (AOC) e o adenoma não é bem esclarecido. Uma hipótese sugere que os ACO alteram os receptores esteroides dos hepatócitos facilitando o crescimento e desenvolvimento tumoral (LÓPEZ DR, et al., 2005).

Existem 4 subtipos de $\mathrm{AH}$ a depender do genótipo-fenótipo: adenomas inflamatórios, adenomas não classificáveis, adenomas HNF1a e adenomas B-catenina. O AH deve ser sempre diferenciado da Hiperplasia Nodular Focal (HNF) sendo que esta representa uma das principais lesões benignas hepáticas, possuindo como característica marcante a presença de cicatriz fibrosa central. Além disso, a HNF possui menor risco de se transformar em neoplasia (PINTO P, 2012).

O tratamento desse tumor benigno pode ser tanto por seguimento clínico, quanto pela realização de procedimento cirúrgico, a depender do tamanho, localização, presença ou não de sintomas. Sintomas como hemorragia intra-abdominal, peritonite, hipotensão e choque hipovolêmico requerem tratamento cirúrgico imediato (CUERVO C, et al., 2014).

O objetivo deste trabalho foi elucidar aspectos fisiológicos, diagnósticos e terapêuticos de uma doença rara, com base em um relato de caso, para que seja feito diagnóstico precoce e evite complicações que possam ser fatais, diminuindo assim, a morbimortalidade. 


\section{DETALHAMENTO DO CASO}

Para ilustrar o relato de caso a seguir, foram solicitadas fotos dos exames de imagem e do pós-operatório da paciente. A mesma autorizou por meio da assinatura do Termo de Consentimento Livre e Esclarecido.

Paciente do sexo feminino, 31 anos, hígida, em uso de anticoncepcional oral combinado por tempo prolongado, assintomática, realizou Ultrassonografia (USG) de abdome total de rotina no dia 10/9/2010, no qual foi evidenciado um nódulo sólido isoecoico a levemente hipoecoico, de contorno regular, medindo $8,1 \mathrm{x}$ $7,1 \times 4,9 \mathrm{~cm}(\mathrm{~T} \times \mathrm{L} \times \mathrm{AP})$ localizado inferiormente ao segmento VI do fígado.

Para elucidação diagnóstica, foi feita Tomografia Computadorizada de abdome com contraste que demonstrou que a massa possuía densidade de partes moles similar ao restante do órgão. Identificou também um ramo da artéria hepática direita como principal nutridor da lesão. Enquanto que a drenagem venosa era feita por tributária que desagua na veia hepática direita. Os exames laboratoriais séricos, incluindo os hepáticos como Transaminase Oxalacética (TGO), Transaminase Pirúvica (TGP) e os de vias biliares, como Fosfatase Alcalina (FA) e Gama Glutamil Transferase (GGT), estavam todos dentro da normalidade.

Por se tratar de um método mais eficaz, foi solicitada Ressonância Magnética (RM) abdominal em que foi vista lesão expansiva dominante de comportamento exofítico no segmento VI com localização periférica, subcapsular. Foi sugerida a presença de tênue gordura no interior da lesão, ausência de sangramento ou de cicatriz central clássica. Com o contraste, a lesão se apresentou hipervascular. Somado-se a essa dominante, havia múltiplas menores com o mesmo aspecto.

De acordo com a Figura 1, a Tomografia Computadorizada de abdome com contraste feita no dia 13/12/2010 no pré-operatório evidencia massa de tamanho consideravelmente aumentado no qual houve impregnação de contraste. Isso corrobora com a hipótese de Adenoma Hepático no qual há aumento de vascularização.

Figura 1 - Exame de imagem abdominal no pré-operatório pós contraste.

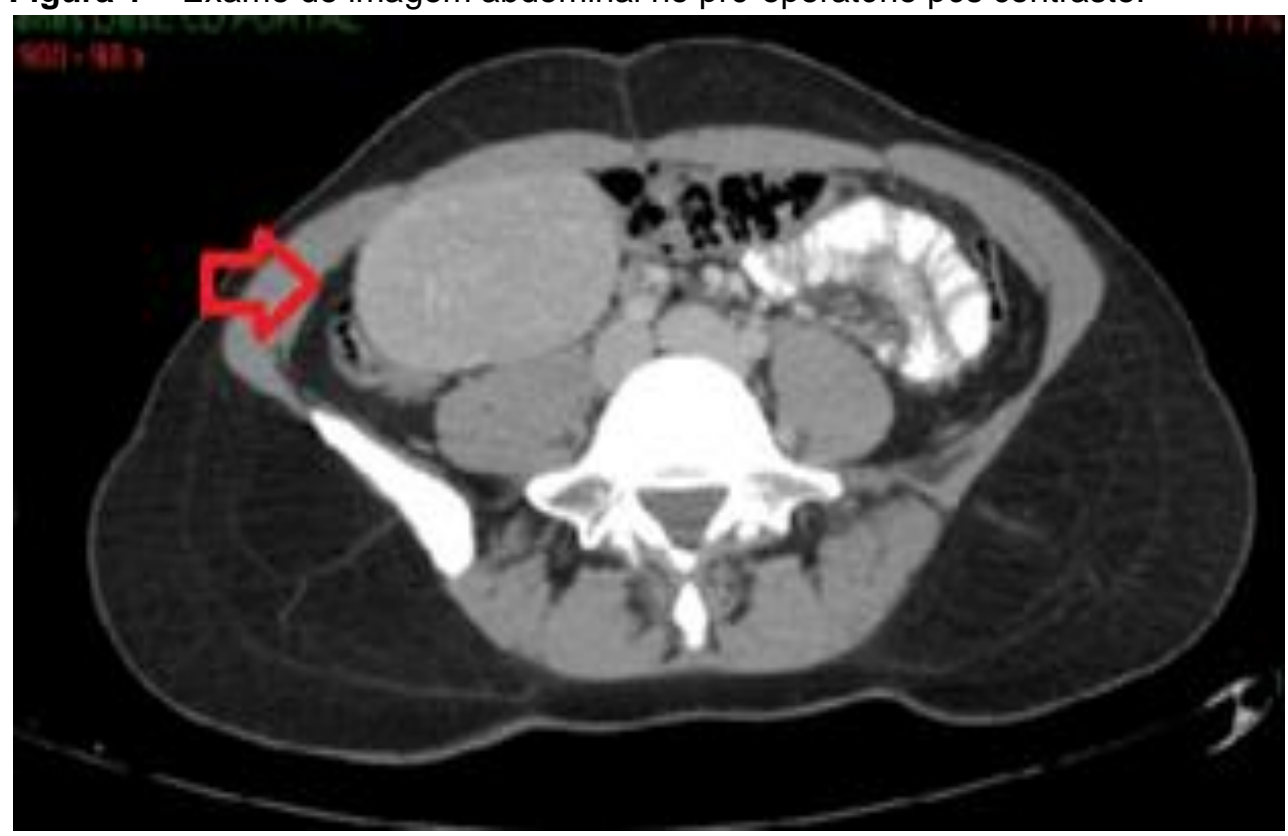

Fonte: Atalla VM, et al., 2021.

De acordo com a Figura 2, na Tomografia Computadorizada de abdome com contraste pré-operatória, realizada no dia 13/12/2010, é possível detectar massa hepática hipervascular medindo $7,9 \mathrm{~cm}$ no seu maior diâmetro. O tumor, de característica regular e circular, se encontra localizado inferiormente no segmento VI do fígado. 
Figura 2 - Exame de imagem abdominal no pré-operatório.

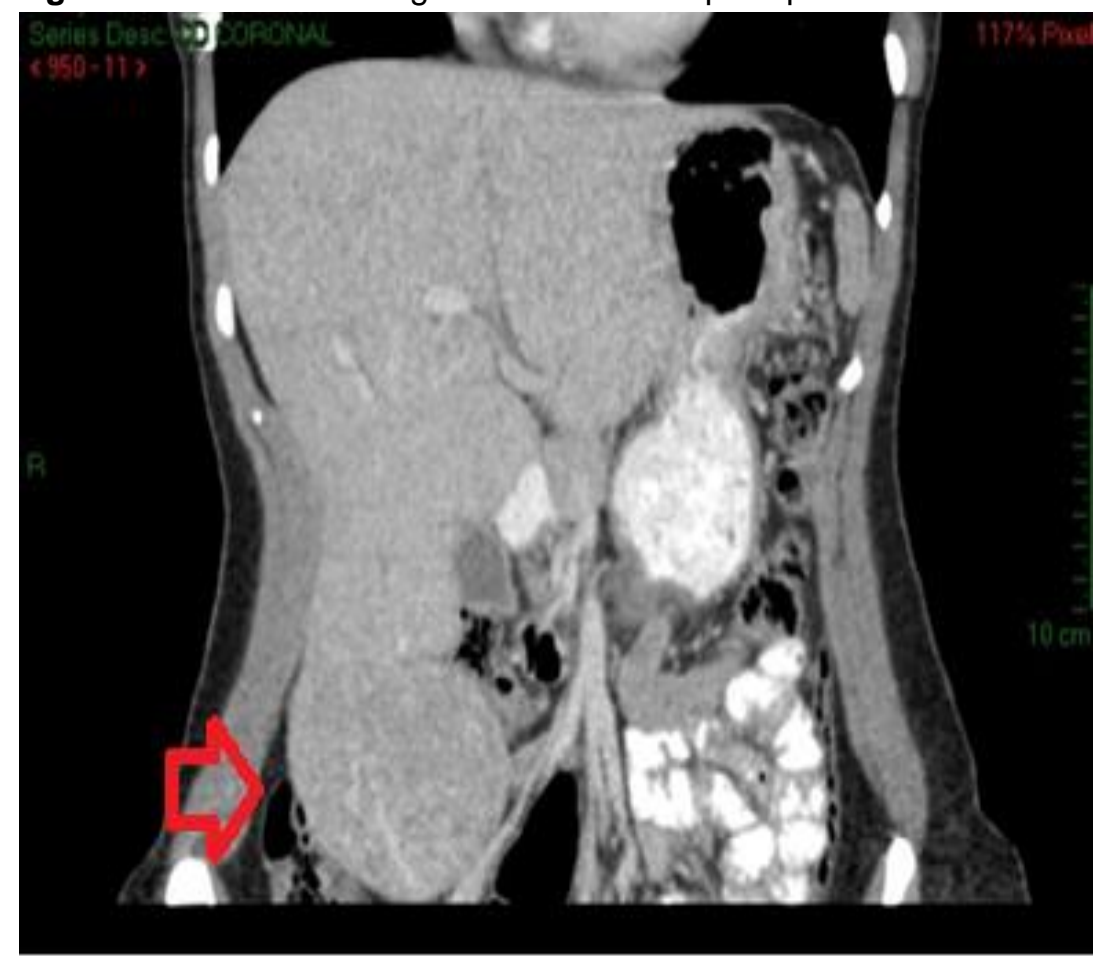

Fonte: Atalla VM, et al., 2021.

Esses achados nos exames em associação com idade, sexo, medicações usadas (AOC) e crescimento evolutivo sugeriram fortemente o diagnóstico de Adenoma Hepático. Em primeiro lugar, foi necessário suspender o uso do anticoncepcional oral tornando contraindicado pelo risco de agravamento das lesões e posterior complicações.

De forma complementar, em decorrência do tamanho do tumor, foi proposto a resolução cirúrgica para o maior (segmentectomia hepática por via aberta) e para os outros menores, controle evolutivo semestral por Ressonância Magnética, passando a anual após a estabilidade dos nódulos em pelo menos dois exames consecutivos.

A cirurgia foi realizada no dia 7/3/2011 com posterior biópsia do material ressecado. Á biópsia, macroscopicamente, a lesão nodular media $8,0 \times 7,0 \mathrm{~cm}$, de aspecto ora pardo amarelado e homogêneo, de consistência firme, ora rosado e amolecido. Microscopicamente, consistia em adenoma hepatocelular exibindo esteatose macro e microgoticular em aproximadamente $20 \%$ da área total avaliada.

Após a cirurgia, para controle de evolução, a paciente fez Ressonância Magnética anualmente em que foi demonstrado nódulos adicionais em diversos segmentos hepáticos, dentre eles no IVB (1 nódulo), VIII e V (5 nódulos), II e III (2 nódulos), os maiores medindo 1,2 e $1 \mathrm{~cm}$.

O exame feito em 2017 mostrou lesão na transição dos segmentos $V$ e VI medindo $0,9 \mathrm{~cm}$ com características de possível Hemangioma Hepático. Em 2020, a Tomografia Computadorizada de abdome e pelve confirmou a presença do Hemangioma e de outras duas imagens noduliformes hipodensas, sem impregnação pelo meio de contraste, medindo cerca de 6 e $14 \times 19$ mm no segmento II.

De acordo com a Figura 3, a partir da Tomografia Computadorizada de abdome realizada no dia 6/1/2020, é possível notar a presença de fígado com contorno característico de ressecção segmentar. Esta imagem reforça que foi retirado todo o tumor que estava presente no segmento VI e o seguimento com exames de imagens demonstra que não houve recidivas. 
Figura 3 - Exame de imagem abdominal no pós-operatório.

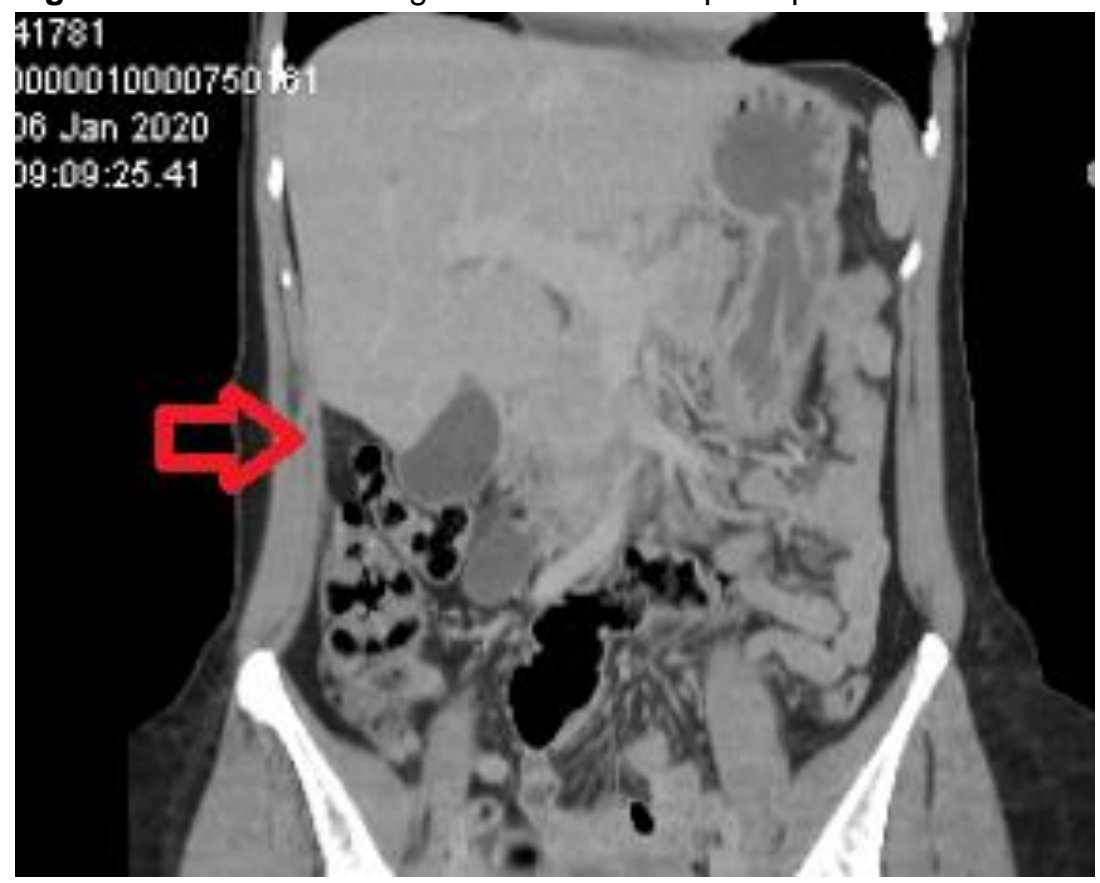

Fonte: Atalla VM, et al., 2021.

De acordo com a Figura 4, mediante autorização da paciente, em consulta de seguimento, foi retirada foto de seu abdome no qual pode-se notar o local em que houve a incisão cirúrgica para retirada da massa hepática. Devido ao porte da cirurgia e aos riscos de possíveis complicações, optou-se por uma incisão maior para que se pudesse ter total controle sobre o ato operatório.

Figura 4 -Cicatriz pós ressecção.

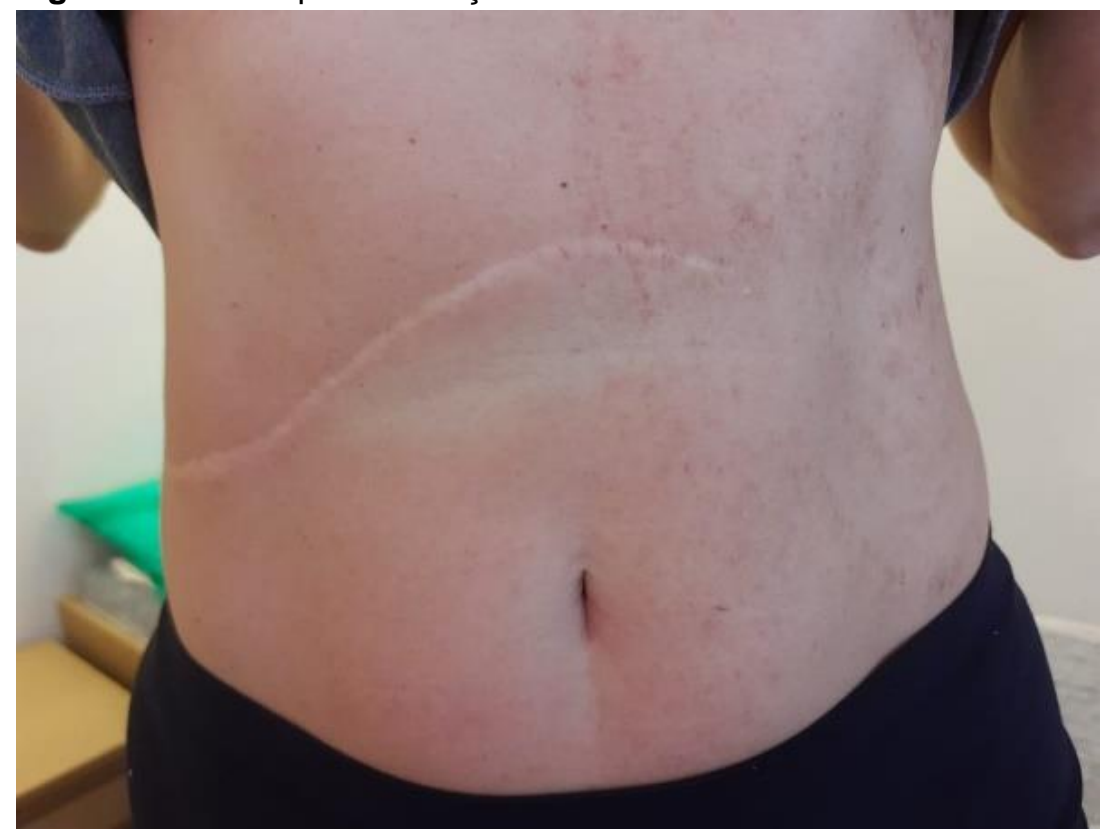

Fonte: Atalla VM, et al., 2021.

A paciente segue assintomática, fazendo o acompanhamento com exames de imagens periódicos anualmente para detecção de possíveis novas lesões e do padrão de evolução das já presentes, que permanecem estáveis. 


\section{DISCUSSÃO}

Os adenomas são formados a partir de grandes cordões celulares constituídos por hepatócitos aparentemente normais, separados por sinusóides dilatados que possuem perfusão arterial, tornando-se hipervasculares nos exames de imagem. Microscopicamente, as células tumorais são constituídas por grande quantidade de glicogênio e lipídios (TIFERES DA e D'IPPOLITO G, 2008). Os tumores podem se apresentar de forma isolada ou em maior número, sendo que se houver mais de 10 lesões, é denominado de Adenomatose Hepática (STRAUSS E, et al., 2015).

Estudos determinaram que existe uma relação restrita entre o uso de anticoncepcionais orais e o desenvolvimento dos adenomas hepáticos. O uso por tempo prolongado e a dose elevada aumentaria a ocorrência, o crescimento em número e tamanho desses tumores (SZOR DJ, et al., 2013). A incidência tende a ser maior nas mulheres por volta 30 anos que usaram AOC por mais de dois anos (PALOMO SANCHEZ JC, et al., 2004).

O AH do tipo inflamatório, possui incidência entre $45-60 \%$, acometendo indivíduos na média entre 12 e 39 anos, predominante no sexo feminino. Como fatores de risco, pode-se citar álcool, anticoncepcional oral e obesidade. Além disso, possui potencial de transformação maligna de 13\%, risco este que aumenta quando há associação com a ativação da ß-catenina. Na histopatologia, ocorre reação inflamatória rica em linfócitos T (CD3) e linfócitos B (CD20) com histiócitos, dilatação sinusoidal, reação ductular e vasos distróficos com bordos bem definidos. Em comparação, o adenoma com inativação do HNF1a, possui incidência entre 35 $40 \%$, acometendo indivíduos na média entre 12 e 37 anos, podendo surgir em crianças se a transmissão for de origem familiar. Ocorre de forma praticamente exclusiva no sexo feminino tendo como principal fator de risco o uso de AOC. Possui potencial de transformação maligna de $7 \%$ e histologicamente, há esteatose marcada sem inflamação ou atipia citológica e com bordos irregulares (PINTO P, 2012).

Em comparação, o AH com ativação da ß-catenina possui incidência de 10 - 15\%, com predominância média entre 16 e 32 anos. Embora mais comum no sexo feminino, é o tipo que mais apresenta componente masculino afetado. Há como fatores de risco, o uso de androgênios exógenos, glicogenoses e Polipose Adenomatosa Familiar, tendo risco de transformação maligna de 46\%. Como histopatologia, há atipia citológica e arquitetura pseudo-acinar. E por último, temos os não classificáveis com incidência de apenas 10\% (PINTO P, 2012).

O diagnóstico normalmente é feito de forma incidental em pacientes assintomáticos que fazem exames complementares, dentre eles, Ultrassonografia de abdome de rotina. Outra forma de se apresentar, seria de forma sintomática, com queixas como dor abdominal localizada em epigástrio e hipocôndrio direito (COELHO JCU, et al., 2011). Além disso, tem a forma mais grave, que cursa com hemorragia intratumoral decorrente da ruptura do mesmo podendo levar a hipotensão e choque hemorrágico (TIFERES DA e D'IPPOLITO G, 2008).

Os exames laboratoriais como alfafetoproteína e função hepática (TGO e TGP) costumam estar normais (TIFERES DA e D'IPPOLITO G, 2008). No caso de grandes adenomas, pode ocorrer aumento das enzimas Fosfatase Alcalina (FA) e Gama Glutamil Transferase (GGT) devido à compressão extrínseca da árvore biliar (PALOMO SÁNCHEZ JC, et al., 2004).

Os exames de imagem de eleição são a Ressonância Magnética (RM) de abdome com contraste e a Tomografia Computadorizada Helicoidal multifásica. A RM consiste em um excelente método, pois, é possível a utilização de meio de contraste hepatobiliar específico favorecendo um diagnóstico correto. Quando realizados, normalmente, é identificada uma lesão sólida com contorno regular, vascularizada e com densidade heterogênea devido à gordura ou ao sangue intratumoral. O diagnóstico definitivo é feito por meio de biópsia e estudo anatomopatológico que se faz em amostra do adenoma ressecado na cirurgia (CUERVO C, et al., 2014).

A hemorragia consiste em uma das complicações desse tumor e esse risco se acentua nos casos de gravidez, lesões grandes, múltiplas, de localização subcapsular e quando há história de uso extenso de anticoncepcionais orais (COELHO JCU, et al., 2011). Outra complicação é a malignização do tumor com o 
desenvolvimento de hepatocarcinoma em que pode haver aumento dos níveis de alfafetoproteína e das dimensões do tumor (TiFERES DA e D'IPPOLITO G, 2008).

O tratamento inicial consiste em suspensão de anabólicos esteroides e anticoncepcionais hormonais, o que pode contribuir para a estabilização do crescimento do tumor ou mesmo redução do seu tamanho. Lesões $<5 \mathrm{~cm}$ assintomática pode-se optar pelo tratamento conservador com acompanhamento regular, enquanto que os tumores $>5 \mathrm{~cm}$ devem ser ressecados pelo risco de ruptura e sangramento (CUERVO C, et al., 2014). Além disso, é indicada cirurgia nos casos de compressão de árvore biliar, pois, isto pode levar a outras condições como icterícia. Lembrando que no tratamento cirúrgico, não há necessidade de ampliar a margem de ressecção desses adenomas (PALOMO SANCHEZ JC, et al., 2004).

Em casos de adenomas não rotos, que não impactem na qualidade de vida e não apresentem característica de malignidade, há técnicas como a embolização arterial ou ablação por radiofrequência que são minimamente invasivas e estão em desenvolvimento constante. Este avanço de tecnologia se dá, pois, reduzem a morbimortalidade relacionada a procedimentos cirúrgicos (NASSER F, et al., 2013). Nos casos de múltiplas lesões não tratáveis por ressecção parcial do fígado, a solução se encontra no transplante hepático.

Os pacientes que realizam o tratamento conservador devem realizar Ultrassonografia ou Tomografia Computadorizada de controle de forma rigorosa a cada seis meses (PAIS-COSTA SR, et al., 2012). Além disso, é recomendado o seguimento por imagem mesmo dos pacientes que foram submetidos à ressecção pelo risco de recidiva. No caso de pacientes com diagnóstico de adenoma hepático e que queiram engravidar, recomenda-se esperar a regressão ou ressecção do mesmo devido ao risco de hemorragia (ROSALES J, et al., 2001). Em pacientes jovens, temos como diagnósticos diferenciais desse tipo de tumor hepático hipervascular a hiperplasia nodular focal, hepatocarcinoma fibrolamelar e metástases (TIFERES DA e D'IPPOLITO G, 2008).

$\mathrm{O}$ AH consiste em uma doença benigna rara, predominante em mulheres em idade fértil e usuária de AOC por período prolongado. Pode ser diagnosticado de forma incidental por meio de exame de imagem e através de sintomas que pode ir de dor abdominal a hemorragia intraperitoneal. O diagnóstico se baseia na biópsia e o tratamento depende do tamanho tumoral, dos sintomas, da localização, podendo ser conservador ou cirúrgico. É imprescindível o seguimento com exames de imagem. O uso de AOC e de androgênios exógenos se tornam contraindicados. A análise do relato de caso e de suas possíveis complicações é fundamental para o aprimoramento do conhecimento médico acerca da patologia para que possa ser realizado o diagnóstico precoce e terapêutica adequada visando reduzir complicações e a morbimortalidade.

\section{REFERÊNCIAS}

1. COELHO JCU, et al. Indicação e tratamento dos tumores benignos do fígado. Arq. Bras. Cir. Dig. (ABCD), 2011; 24(4): 318-323.

2. COSTA SRP, et al. Setorectomia posterior direita laparoscópica no tratamento dos tumores hepáticos. Arq. Bras. Cir. Dig. (ABCD), 2010; 23(4):275-279.

3. CUERVO C, et al. Hepatocellular Adenomas: Current Findings in Images Which Allow their Characterization and Management. Revista Colombiana de Radiologia, 2014; 25(2): 3934-41.

4. GONCALVES DL, et al. Hepatic Adenomatosis: A Challenging Liver Disease. Portuguese Journal Gastroenteroly, 2020; 27(1): 37-42.

5. HERMAN P, et al. Ressecões hepáticas por videolaparoscopia. Arq. Bras. Cir. Dig. (ABCD), 2009; 22(4):226-32.

6. LÓPEZ DR, et al. Hepatic adenoma related to oral contraceptives use. Atencion primaria, 2005; 35(2):109-110.

7. NASSER F, et al. Minimally invasive treatment of hepatic adenoma in special cases. Einstein, 2013; 11(4):524-7.

8. NUNES TF, et al. Pyogenic liver abscess as a late complication after embolization of a hepatic adenoma. Radiologia brasileira, 2019; 52(2):134-135.

9. PAIS-COSTA SR, et al. Adenoma hepático gigante associado com uso abusivo de esteróide androgênico anabolizante: relato de caso. Arq. Bras. Cir. Dig. (ABCD), 2012; 25(3):180-182.

10. PALOMO SÁNCHEZ J. C, et al. Adenoma hepático. Oncologia, 2004; 27(5): 45-48.

11. PINTO LEV, et al. Late surgical treatment for spontaneous rupture of hepatocellular adenoma: case report. Arq. Bras. Cir. Dig. (ABCD), 2015; 28(4):297- 299. 
12. PINTO P. Adenomas hepáticos: do genótipo à clínica. Arquivos de Medicina, 2012; 26(2): 69-77.

13. RODRIGUES TFC, et al. Hepatectomia aberta, videolaparoscópica e assistida por robótica em ressecção de tumores hepáticos: uma revisão não sistemática. Arq. Bras. Cir. Dig. (ABCD), 2017; 30(2):155-160.

14. ROSALES J, et al. Ruptura de un adenoma hepático como causa de sangrado en el tercer trimestre de la gestación: reporte de un caso y discusión de la literatura. Revista de Gastroenterología, 2001; 21(4): 312-315.

15. STRAUSS E, et al. Diagnosis and treatment of benign liver nodules: Brazilian Society of Hepatology (SBH) recommendations. Arquivos de Gastroenterologia, 2015; 52(1): 47-54.

16. SZOR DJ, et al. Adenoma hepático. Arq. Bras. Cir. Dig. (ABCD), 2013; 26(3):219-222.

17. TIFERES DA, D'IPPOLITO G. Neoplasias hepáticas: caracterização por métodos de imagem. Radiologia brasileira, 2008; 41(2):119-127. 